University of Tennessee Health Science Center

UTHSC Digital Commons

\title{
Conditional Loss of Engrailed 1/2 in Rhombic Lip-Derived Neurons Increases Intrinsic Rhythmicity and Decreases Overall Variability of Eupneic Respiration
}

Angela P. Taylor

University of Tennessee Health Science Center

Follow this and additional works at: https://dc.uthsc.edu/dissertations

Part of the Medical Cell Biology Commons, Medical Neurobiology Commons, Medical Physiology Commons, Neurosciences Commons, and the Physiological Processes Commons

\section{Recommended Citation}

Taylor, Angela P. (https://orcid.org/0000-0001-5391-607X), "Conditional Loss of Engrailed 1/2 in Rhombic Lip-Derived Neurons Increases Intrinsic Rhythmicity and Decreases Overall Variability of Eupneic Respiration" (2020). Theses and Dissertations (ETD). Paper 541. http://dx.doi.org/10.21007/ etd.cghs.2020.0528.

This Thesis is brought to you for free and open access by the College of Graduate Health Sciences at UTHSC Digital Commons. It has been accepted for inclusion in Theses and Dissertations (ETD) by an authorized administrator of UTHSC Digital Commons. For more information, please contact jwelch30@uthsc.edu. 


\title{
Conditional Loss of Engrailed 1/2 in Rhombic Lip-Derived Neurons Increases Intrinsic Rhythmicity and Decreases Overall Variability of Eupneic Respiration
}

\begin{abstract}
Evidence for a cerebellar role during cardiopulmonary challenges has long been established, but investigation into cerebellar involvement in eupneic breathing has been inconclusive. Given the view of the cerebellum (CRB) as a temporally coordinating structure, any investigation into the CRB during respiration must evaluate rhythm and variability of the respiratory sequence. In this study, we chose an elegant model of cerebellar neuropathology, Atoh1-En1/2 CKO, where mutant animals have conditional loss of the developmental patterning gene Engrailed 1/2 in rhombic lip-lineage neurons and exhibit a proportional scaling-down of neuron number in hypoplastic lobules of the CRB. We utilized whole-body unrestrained plethysmography to measure respiration during eupnea, and evaluated the respiratory sequence of mutant animals and their control littermates using the average rate, the coefficient of variation (CV), and a unique measure of intrinsic rhythmicity called CV2. Linear regression analyses revealed that mutant animals had decreased overall variability and increased intrinsic rhythmicity (as measured by CV and CV2, respectively) compared to their control littermates, but we found no effect of strain on average respiratory rate. Analysis also revealed modestly decreased respiratory rates, increased $\mathrm{CV}$, and increased CV2 in female animals, independent of strain. These results align well with previously reported studies and add new insight into CRB involvement in eupneic respiratory rhythmicity. Although not investigated in this study, future works should consider the coordination of breathing, licking, swallowing, and whisking in order to build a more complete understanding of the relationship between the CRB and respiration.
\end{abstract}

\section{Document Type}

Thesis

\section{Degree Name}

Master of Science (MS)

\section{Program}

Biomedical Sciences

Research Advisor

Detlef H. Heck, PhD

\section{Keywords}

breathing, cerebellum, deep cerebellar nuclei, engrailed, mouse, respiration

\section{Subject Categories}

Medical Cell Biology | Medical Neurobiology | Medical Physiology | Medical Sciences | Medicine and Health Sciences | Neurosciences | Physiological Processes 
University of Tennessee HeAlth Science Center

MASTER OF SCIENCE THESIS

\section{Conditional Loss of Engrailed 1/2 in Rhombic Lip-Derived Neurons Increases Intrinsic Rhythmicity and Decreases Overall Variability of Eupneic Respiration}

Author:

Angela P. Taylor
Advisor:

Detlef H. Heck, PhD

A Thesis Presented for The Graduate Studies Council of

The University of Tennessee Health Science Center

in Partial Fulfillment of Requirements for the Master of Science degree from

The University of Tennessee

in

Biomedical Sciences: Neuroscience

College of Graduate Health Sciences

September 2020 
Copyright (C) 2020 by Angela P. Taylor.

All rights reserved.

Modified with permission Masters/Doctoral Thesis LaTeX Template

Version $2.5(8 / 27 / 2017)$

http://www.LaTeXTemplates.com Creative Commons License CC BY-NC-SA 3.0 
This work, as in all my life, is dedicated to my Creator. "And whatever you do, in word or deed, do everything in the name of the Lord Jesus, giving thanks to God the Father through him." Colossians 3:17 ESV 


\section{Acknowledgements}

First, I would like to sincerely thank my mentor Dr. Detlef H. Heck for his wisdom, dedication, and patience in training me to be an independent researcher. I would also like to thank my committee members past and present, Dr. Victor V. Chizhikov, Dr. Matthew Ennis, Dr. Monica M. Jablonski, and Dr. Michael P. McDonald. All have encouraged and guided me along the way, and I am grateful for their generosity in sharing their time and experience. I would also like to thank Dr. Max Fletcher, Dr. Joseph Callaway, Dr. Elizabeth Fitzpatrick, Dr. Donald Thomason, Dr. Isaac Donkor, and Dr. John Cox for providing mentorship, wisdom, and many opportunities to grow as a professional.

I would like to thank Dr. Alexandra Joyner at Memorial Sloan Kettering Cancer Center for kindly providing the study animals for this work and sharing her expertise. I would also like to acknowledge Brittany Correia, for her assistance with data collection, and for her encouragement, camaraderie, and humor; Dr. Liu Yu, for training me in countless laboratory techniques and analyses, providing sound advice with endless patience; and Shuhua Qi, for her kindness and assistance with all manner of laboratory needs. I would also like to acknowledge Patricia Goedecke and Dr. Elizabeth A. Tolley from the Biostatistics, Epidemiology, and Research Design Unit for their assistance in interpreting the linear regression results in this work. Next, I would like to acknowledge the Neuroscience Institute at the University of Tennessee Health Science Center for financial support, and the National Institute of Mental Health of the National Institutes of Health for support under award numbers R01MH112143, R01MH112143-02S1, R37MH085726, and F31MH122068. The content is solely the responsibility of the author and does not necessarily represent the official views of the National Institutes of Health.

To my fellow graduate students, my friends, my church family - I struggle to articulate the impact you have had on me during this very difficult but rewarding process. You have provided counsel when asked, distraction when needed, and always friendship. From the bottom of my heart, thank you, and I will always be here to return the favor. To my family - none of this would have been possible without your commitment to my education as a child and support as an adult. Thank you for your sacrifices, your words of encouragement, and your prayers. Finally and in everything, I gratefully acknowledge my savior Jesus for his faithfulness, strength, and sustaining grace. "In their hearts humans plan their course, but the Lord establishes their steps." Proverbs 16:9 NIV 


\section{Abstract}

Angela P. Taylor

Conditional Loss of Engrailed 1/2 in Rhombic Lip-Derived Neurons

Increases Intrinsic Rhythmicity and Decreases Overall Variability of

Eupneic Respiration

Evidence for a cerebellar role during cardiopulmonary challenges has long been established, but investigation into cerebellar involvement in eupneic breathing has been inconclusive. Given the view of the cerebellum (CRB) as a temporally coordinating structure, any investigation into the CRB during respiration must evaluate rhythm and variability of the respiratory sequence. In this study, we chose an elegant model of cerebellar neuropathology, Atoh1-En1/2 CKO, where mutant animals have conditional loss of the developmental patterning gene Engrailed 1/2 in rhombic lip-lineage neurons and exhibit a proportional scaling-down of neuron number in hypoplastic lobules of the CRB. We utilized whole-body unrestrained plethysmography to measure respiration during eupnea, and evaluated the respiratory sequence of mutant animals and their control littermates using the average rate, the coefficient of variation $(\mathrm{CV})$, and a unique measure of intrinsic rhythmicity called CV2. Linear regression analyses revealed that mutant animals had decreased overall variability and increased intrinsic rhythmicity (as measured by CV and $\mathrm{CV} 2$, respectively) compared to their control littermates, but we found no effect of strain on average respiratory rate. Analysis also revealed modestly decreased respiratory rates, increased CV, and increased CV2 in female animals, independent of strain. These results align well with previously reported studies and add new insight into CRB involvement in eupneic respiratory rhythmicity. Although not investigated in this study, future works should consider the coordination of breathing, licking, swallowing, and whisking in order to build a more complete understanding of the relationship between the CRB and respiration. 


\section{Contents}

1 Introduction $\quad 1$

1.1 Evidence for Cerebellar Involvement in Respiratory Function . . . . . . . . 2

1.2 The Cerebellum as Timekeeper and the Cerebellar Microcircuit . . . . . . . 3

1.3 Development and Characteristics of the Atoh1-En1/2 CKO Strain . . . . . . . 4

2 Materials and Methods 6

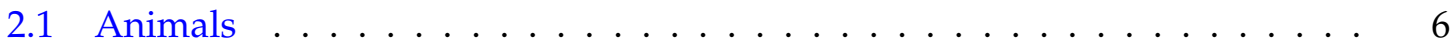

2.2 Plethysmograph Recording . . . . . . . . . . . . . . . . 7

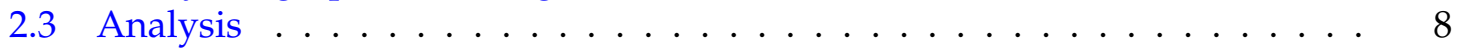

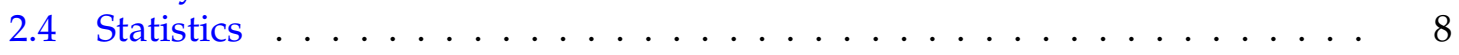

3 Results 9

3.1 Conditional Loss of En1/2 in Excitatory Cerebellar Neurons Has No Effect on Respiratory Rate . . . . . . . . . . . . . . . . . . . . . 9 9

3.2 Mice with Conditional Loss of En1/2 in Excitatory Cerebellar Neurons Show Decreased Overall Respiratory Variability . . . . . . . . . . . . . . . 12

3.3 Mice with Conditional Loss of En1/2 in Excitatory Cerebellar Neurons Show Increased Intrinsic Respiratory Rhythmicity . . . . . . . . . . . . . . 12

4 Discussion $\quad 15$

$\begin{array}{ll}\text { List of References } & 18\end{array}$

$\begin{array}{ll}\text { Vita } & 23\end{array}$ 


\section{List of Tables}

2.1 Mouse group composition and means . . . . . . . . . . . . . 6 


\section{List of Figures}

2.1 Whole-body unrestrained plethysmograph recording $\ldots \ldots \ldots \ldots$

3.1 IRI data do not fit assumptions of linear regression . . . . . . . . . . . . . 10

3.2 Average respiratory rate is influenced by sex and not strain . . . . . . . . . 11

3.3 CV of IRI distribution is influenced by both sex and strain . . . . . . . . . . 13

3.4 Mean CV2 is influenced by both sex and strain . . . . . . . . . . . . . . . 14 


\title{
List of Abbreviations
}

\author{
Atoh1 Atonal homolog 1 \\ $\mathrm{CKO}$ Conditional knockout \\ CRB Cerebellum \\ $\mathrm{CV}$ Coefficient of variation \\ DCN Deep cerebellar nuclei \\ eCN Excitatory cerebellar nuclei neurons \\ En1/2 Engrailed 1/2 \\ IRI Inter-respiratory interval \\ mDCN Medial deep cerebellar nucleus \\ PC Purkinje cell
}




\section{Chapter 1}

\section{Introduction}

The involvement of the cerebellum (CRB) in cardiopulmonary function such as blood pressure regulation and respiration has long been established and is evidenced by a wealth of anatomical and neuropathological data $[2,40,23,16,57,19,9,31,13,43]$. While many have supported CRB involvement in breathing during respiratory challenges, like hunger for air and hypoxia, a similar relationship has not been found when evaluating normal breathing, also called eupneic breathing or eupnea [35, 61, 39, 31, 8]. This study seeks to investigate the cerebellar role during eupneic respiration using a mouse model of abnormal cerebellar development. This model, with conditional loss of the developmental patterning genes Engrailed 1 and $2(E n 1 / 2)$ in rhombic lip-derived neurons, presents a great opportunity to further our understanding of this cerebellar role. Mutant animals have demonstrated motor deficits, a proportional scaling down of neuron number in hypoplastic lobules of the $\mathrm{CRB}$, and significantly reduced neuron number in the medial and intermediate deep cerebellar nuclei $(\mathrm{DCN})[53,38]$, but no studies to date have evaluated the effect of the conditional loss of $E n 1 / 2$ on eupneic breathing in these animals.

Many studies which have failed to implicate the CRB during eupneic respiration have not evaluated changes to the respiratory pattern. Therefore, our lab recently investigated changes to respiratory patterns during eupneic breathing using a mouse model with Purkinje cell (PC) silencing [28]. Unlike other common models of ataxia with broad neuropathology, this mouse model of cerebellar ataxia lacks synaptic vesicle transmission only in PCs, thereby preventing output from the cerebellar cortex [51]. The lack of PC output did not affect the average respiratory rate, as measured by the mean inter-respiratory interval (IRI), nor did it affect the coefficient of variation (CV). While CV is an effective measure of overall variability, it is not sensitive to either brief variability in adjacent respiratory intervals or slow rate changes. CV is calculated from the total IRI distribution, but does not take into account the chronological order of IRIs. To investigate whether this intrinsic rhythmicity was affected, we utilized a measure called CV2, which was first described by Holt et al. in 1996 to analyze intrinsic variability in visual cortex neuron spiking in cats [22]. CV2 is calculated by finding the standard deviation of adjacent IRIs, divid-

ing by their mean, and scaling by $\sqrt{2}$ (Eq. 2.1) [22]. The CV2 of a sequence, then, is the mean of all CV2s calculated for each adjacent pair of IRIs in the sequence. We found that the absence of cerebellar cortical output led to significantly decreased CV2 in mutants as 
compared to control animals, which indicates increased intrinsic respiratory rhythmicity [28]. These novel results confirmed the importance of the cerebellum in coordinating respiratory rhythms during eupneic breathing.

Whereas our previous study emphasized the importance of the cerebellar cortex during eupnea, this study aimed to investigate the role of excitatory cerebellar nuclei $(\mathrm{eCN})$ neurons. The eCN are not only the final output of the cerebellum, but they integrate information from PC as well as climbing fiber and mossy fiber collaterals [41, 45, 26]. Lu et al. has previously shown that there are robust projections from medial DCN $(\mathrm{mDCN})$ and some projections from intermediate $\mathrm{DCN}$ to the ventromedial reticular formation, a structure involved in respiratory and orofacial motor control [30, 44, 7, 12, 63]. In addition, they found that a subset of $\mathrm{mDCN}$ neurons showed firing patterns which were significantly correlated with respiration [30]. Therefore, this present study used a unique mouse model developed by Orvis et al. [38] with conditional loss of function of the developmental patterning genes Engrailed 1 and 2 (En1/2) in excitatory cerebellar neurons. This model (Atoh1-En1/2 CKO) is especially appropriate in that normal cerebellar cytoarchitecture is preserved, with only proportional scaling down of neuron number in hypoplastic lobules and a reduction of the intermediate and medial DCN [53]. This model has shown deficits in motor behaviors [53], but no studies to date have examined the effect of conditional loss of $E n 1 / 2$ in excitatory cerebellar neurons on respiratory rhythmicity. Given the results from our recent study and our understanding of the Atoh1-En1/2 CKO mouse model, we hypothesized that conditional loss of $E n$ 1/2 in excitatory cerebellar neurons would result in increased intrinsic rhythmicity (CV2), but no change in overall variability $(\mathrm{CV})$ or respiratory rate.

\subsection{Evidence for Cerebellar Involvement in Respiratory Function}

Regions associated with respiratory pattern generation are primarily located in the brainstem, including the pre-Bötzinger complex, Bötzinger complex, retrotrapezoid nucleus, and the rostral, caudal and pontine respiratory groups [13]. Although most early research involving the cerebellum focused on its role in voluntary functions [34], scientists have also investigated a putative role in autonomic functions such as respiration [35]. The mechanism of involvement is still being elucidated, but there is growing electrophysiological, anatomical, and functional evidence to support a cerebellar role in breathing. specifically pointing to the $\mathrm{mDCN}$. In a 1992 study using alert cats, Gruart and Delgado-Garcia recorded neurons in the $\mathrm{mDCN}$ which were temporally aligned with eupneic respiration [17]. In addition, several studies found that stimulating the $\mathrm{mDCN}$ modulated phrenic nerve activity and respiratory rate $[54,58,59]$. Lu et al. further supported $\mathrm{mDCN}$ involvement, recording $\mathrm{mDCN}$ single unit activity which was correlated with respiration [30]. Using retrograde and anterograde tract tracing techniques, their study also found projections from the $\mathrm{mDCN}$ and intermediate $\mathrm{DCN}$ to brainstem regions containing respiratory pattern generators, specifically the ventromedial medullary reticular formation. 
In addition, the cerebellum has been implicated in several studies investigating cardiopulmonary function. Harper et al. [19] used functional magnetic resonance imaging to identify structures that become differentially activated (as compared to baseline) during cardiopulmonary challenges. Specifically, they found that inspiratory loading and hypercapnic stimulation resulted in recruitment of the deep cerebellar nuclei and ventral cerebellum, respectively. Others have reported cerebellar activation during procedures which increased blood pressure, hypercapnia, and expiratory loading [2, 23, 16, 57]. While there is a clear involvement of the cerebellum during cardiopulmonary challenges, these studies have failed to implicate the cerebellum during eupnea [35, 61, 39, 60, 31, 8]. One study by $\mathrm{Xu}$ and Frazier investigated respiratory variables such as tidal volume in anesthetized cats, comparing normal animals to cats either post-cerebellectomy or with thermal lesions of the mDCN [57]. Progressive and transient hypoxia revealed changes in respiratory variables, but no difference between animals was found during eupneic breathing. This trend has continued even in recent years, as in a 2016 investigation of breathing in a mouse model of cerebellar ataxia called Lurcher, which has total Purkinje cell loss [8]. Again, differences between control and mutant animals were only significant during respiratory challenges.

These studies were valuable in furthering our understanding of cerebellar function as it relates to cardiopulmonary challenges, but they form an incomplete picture of the cerebellar role in eupneic respiration because respiratory sequence variability was not considered. The cerebellum can be viewed as a highly precise temporally coordinating structure, which will be described in greater detail below. Two separate studies, using the same mouse model, discovered that animals without PC transmission have altered eupneic respiratory patterns compared to control littermates [20, 28]. In van der Heijden and Zoghbi, PC silencing resulted in increased inter-breath irregularity during normoxic conditions. Therefore, to fully understand the cerebellar role during eupneic respiration, the finer aspects of respiratory timing must also be evaluated.

\subsection{The Cerebellum as Timekeeper and the Cerebellar Microcircuit}

As our understanding of cerebellar anatomy and physiology has developed, experts have grown to view the cerebellum as a timekeeper, precisely coordinating other structures to facilitate not only fine motor control but also complex cognitive functions [1]. In a recent consensus paper on the subject, Bareš et al. argue that the high density of neurons, strictly organized microcircuitry, and vast interconnectivity with other cortical structures make the cerebellum uniquely suited to this role. Within the scope of this study, the cerebellar microcircuit is of particular importance.

Sensory and postural information from the cortex, spinal cord and vestibular system are routed through the pons and enter the cerebellum as mossy fibers [41]. These mossy fibers synapse primarily with granule cells in the internal granule cell layer, and 
granule cells extend their axons radially to the molecular layer and then bifurcate as parallel fibers [41]. The parallel fibers travel along the mediolateral axis, synapsing with PC dendritic arbors - a single rat PC can receive synaptic connections from about 175,000 parallel fibers [41, 36]. In contrast, climbing fibers form strong connections with 5-10 PCs, with one PC only receiving input from a single climbing fiber [48]. These climbing fibers carry sensorimotor and cognitive information from the inferior olive, and they are responsible for the distinct complex spike that be seen in PC electrophysiological recordings [48]. PCs then form the sole output of the cerebellar cortex and project to the deep cerebellar nuclei, which are made up of inhibitory neurons targeting the inferior olive and the eCN $[15,49]$. The eCN integrate information from not only the PCs but from mossy fiber and climbing fiber collaterals, then exit the cerebellum as its final output to the thalamus and other brainstem areas [41, 45, 26].

It is agreed that the cerebellar microcircuit is essential for cerebellar temporal coordination, but how this role affects eupneic respiration is not well understood. One important study investigated a putative role of the $\mathrm{mDCN}$ in coordinating eupneic respiration with orofacial movements, such as licking and swallowing [30]. In addition to the previously described anatomical evidence, they found a subset of $\mathrm{mDCN}$ neurons with spiking patterns which were significantly correlated with both respiration and swallowing. Furthermore, our research in Liu et al. highlighted the significance of PC output in the intrinsic rhythmicity of breathing [28]. This study then seeks to further our understanding by investigating the $\mathrm{eCN}$ and the effects of their abnormal development on the respiratory rhythm.

\subsection{Development and Characteristics of the Atoh1-En1/2 CKO Strain}

As the final output of the cerebellum, the excitatory cerebellar nuclei (eCN) must be investigated to fully understand the cerebellar role in respiration. To do so, we will utilize a unique mouse model originated by Orvis et al. with conditional loss of the developmental patterning genes Engrailed 1/2 (En1/2) in rhombic lip-derived neurons [38]. These animals were developed using a Cre/lox system which conditionally knocks out En1/2 in excitatory cerebellar neurons, including granule cells and the eCN, using the Atoh1 promoter (Atoh1-En1/2 CKO).

Engrailed 1 and 2 are homeobox transcription factors which have been shown to be essential for normal development of the brain [55, 25,53]. They have overlapping but not identical expression patterns, primarily in the developing midbrain and hindbrain [11, 55]. Mutant mouse strains have revealed that En1/2 are crucial in the CRB for normal molecular patterning of the PC parasagittal stripes, foliation, and afferent circuit maps $[47,10,46]$.

Given the widespread role of En1/2 in the developing brain, we have selected a mouse model which conditionally knocks out En1/2 expression in rhombic lip-derived neurons. The cerebellar rhombic lip and ventricular zone are the complementary progenitor zones which develop into the cerebellum [26]. The ventricular zone progenitors 
give rise to cerebellar GABAergic neurons, including Purkinje cells, inhibitory interneurons, and Bergmann glia [26]. Rhombic lip progenitor cells express the transcription factor Atonal homolog 1 (Atoh1) and give rise to the glutamatergic neurons of the DCN, granule cells, as well as several brainstem nuclei $[32,50]$. Fate mapping studies helped clearly define structures originating from the rhombic lip, and a good summary of these results can be found in Wingate 2005 [56]. Due to the importance of brainstem structures in governing respiration, it is unsurprising that Atoh1-null mice do not survive postnatally due to respiratory failure [24].

In terms of cerebellar size and foliation, the Atoh1-En1/2 CKO strain suffers proportional scaling down of neuron number in hypoplastic lobules of the anterior and central vermis and paravermis, as well as several shortened or missing fissures [38, 53]. In addition, the medial and intermediate deep cerebellar nuclei are greatly reduced in size, yet cerebellar cytoarchitecture is maintained [53]. This crucial aspect of this model sets it apart from other cerebellar mutant strains and makes it ideal for this investigation. Thus the Atoh1-En1/2 CKO strain, with its unique developmental irregularities and preserved cytoarchitecture, lends great potential to this investigation of a cerebellar role in eupneic respiration. 


\section{Chapter 2}

\section{Materials and Methods}

\subsection{Animals}

All animals were bred and genotyped in the lab of Dr. Alexandra Joyner (Memorial Sloan Kettering Cancer Center, USA) and then transferred to the animal care facilities at the University of Tennessee Health Science Center (UTHSC). Use of animals and all experimental procedures were performed under the approval of the Institutional Animal Care and Use Committee of UTHSC. In total, 39 animals were used for analysis (details in Table 2.1).

Table 2.1: Mouse group composition and means.

\begin{tabular}{llll}
\hline Variables & All $(\mathrm{N}=39)$ & Mutant $(\mathrm{N}=19)$ & Control $(\mathrm{N}=20)$ \\
\hline Sex & & & \\
Female & $24(61.54 \%)$ & $12(30.77 \%)$ & $12(30.77 \%)$ \\
Male & $15(38.46 \%)$ & $7(17.95 \%)$ & $8(20.51 \%)$ \\
& & & \\
Mean IRI (s) & & \\
Rate & $0.164(0.035)$ & $0.156(0.030)$ & $0.172(0.039)$ \\
CV & $6.30(1.12)$ & $6.58(1.06)$ & $6.03(1.13)$ \\
CV2 & $0.37(0.06)$ & $0.34(0.07)$ & $0.39(0.06)$ \\
\hline
\end{tabular}

${ }^{\text {a }}$ Count and (\%) for discrete variables

${ }^{\mathrm{b}}$ Mean and (standard deviation) for continuous variables

Originally developed and described by Orvis et al. [38], this strain uses a Cre/lox system and the Atoh1 promoter to conditionally knock out the Engrailed 1/2 genes in rhombic lip-derived excitatory cerebellar neurons. Mutant animals (Atoh1-Cre/+;En1 fx/fx; En2 $f x / f x$ ) were compared to their control littermates $(E n 1 f x / f x ; E n 2 f x / f x)$. We recorded animals at 4-5 months old, corresponding to mature adulthood in mice [14]. 


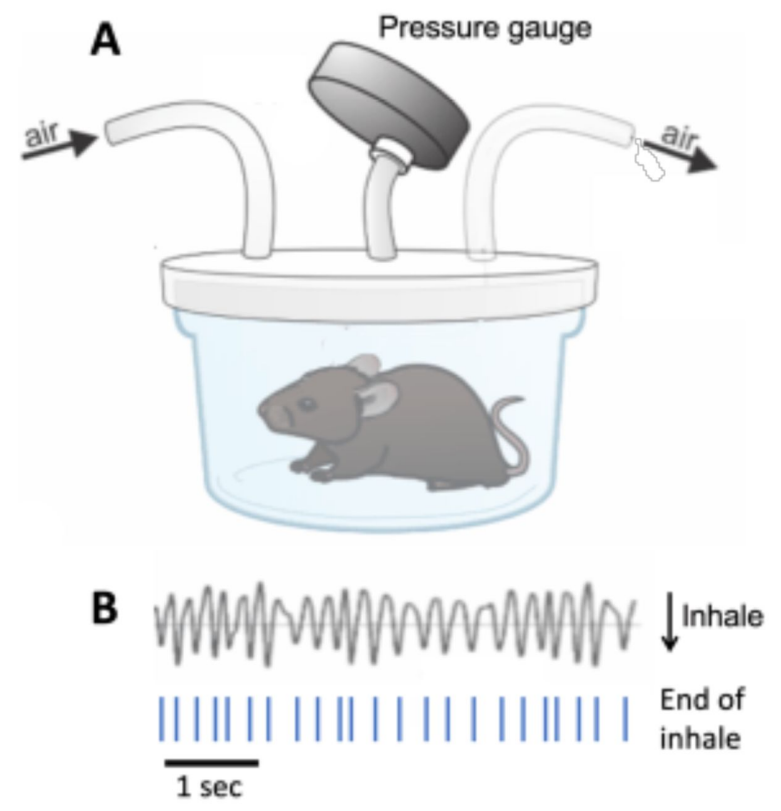

Figure 2.1: Whole-body unrestrained plethysmograph recording. A) Example plethysmograph chamber. Pressure changes due to respiration are reflected by voltage changes, shown in B. B) In this illustration, voltage decreases as pressure increases due to respiration (the reverse is true in the present study - voltage increased with pressure increase.) These pressure increases are then marked, representing the end of inspiration. The sequence of marks is then used for all further analysis. Reprinted with open access permission. Liu et al. [28]

\subsection{Plethysmograph Recording}

To record respiratory patterns under eupneic conditions, the protocol described previously was followed [28]. In short, we used a plethysmograph chamber as shown in Figure 2.1A, constructed using an airtight container with constant air flow and a pressure transducer attached. Animals were recorded individually, an opaque covering was placed over the entire apparatus, and the animal's breathing was recorded for 30 minutes using Spike2 recording software (Cambridge Electronic Design, UK). As the animal inhales, pressure inside the chamber increases slightly, causing the voltage to increase (note: Figure 2.1B shows voltage decreasing with inspiration). The peaks, or end of inspiration, were marked and this sequence was used for all further analysis. This included both normal breathing and sniffing behavior under normoxic conditions. Between recording each animal, the chamber was cleaned with a $70 \%$ ethanol solution and allowed to dry. 


\subsection{Analysis}

In Spike2, the raw signal was first processed using a FIR Butterworth low pass filter with a cutoff at $20 \mathrm{~Hz}$ to remove noise without distorting the respiratory data, as suggested by Lim et al. [27]. To remove very low frequency $(<1 \mathrm{~Hz})$ drift, the built-in Spike2 DC remove channel process was utilized (time constant $=0.1$ seconds). Signal peaks were marked using a minimum interval of 0.06 seconds to exclude local maxima which do not correspond to the end of inspiration, given that murine respiratory rates vary from $1-12 \mathrm{~Hz}$ (including sniffing behavior) [52,51]. Minimum peak height varied based on the amplitude of individual recordings, but ranged from $0.15-0.35$ Volts. Inter-respiratory intervals (IRI) were determined by calculating the time between subsequent end-of-inspiration marks $(\Delta t) . C V$ was calculated as the standard deviation of the IRI distribution divided by the mean. Finally CV2 [22], which compares subsequent IRIs, was calculated using Eq. 2.1.

$$
C V 2=\frac{2\left|\Delta t_{(i+1)}-\Delta t_{(i)}\right|}{\Delta t_{(i+1)}+\Delta t_{(i)}}
$$

To preserve data integrity, it was necessary to exclude from analysis periods of noise, which can be caused by the animal eliminating or by blocking the input or output tubes with its body [27]. For each mouse, the CV, CV2, and mean IRI of five respiratory sequences (each sixty seconds long) were calculated independently and then averaged. All calculations and statistical analyses were performed using MATLAB scripts (MathWorks, USA).

\subsection{Statistics}

First, the response variables were tested to ensure that they were appropriate for linear regression. In the following tests for normality and homoscedasticity, data for each response variable were treated as a single group (for example, all CV data was grouped regardless of sex or strain). First, response variables were evaluated to ensure normality using quantile-quantile (QQ) plots, which compare the distribution of a data set with the normal distribution. The Breusch-Pagan test was used to evaluate the data for homoscedasticity, where the null hypothesis is that data are homoscedastic and a $p$-value $\geq 0.05$ indicates heteroscedasticity $[3,33]$. To determine the effect of sex and strain on response variables (CV, CV2, and mean IRI), linear regression analysis was applied. Models were fitted to relate response variables to predictor variables (sex and strain). Insignificant predictor variables ( $p \geq 0.05$ ) were removed to increase degrees of freedom and improve the model. Model fit was confirmed using residual plots. 


\section{Chapter 3}

\section{Results}

\subsection{Conditional Loss of En1/2 in Excitatory Cerebellar Neurons Has No Effect on Respiratory Rate}

To evaluate whether conditional loss of $E n 1 / 2$ in eCN has an effect on average respiratory rate during eupnea, we intended to use mean IRI as in Liu et al. 2020 [28]. However, the QQ plot of IRI data, as well as the total IRI distribution (including all IRIs instead of averages) both showed clear right skews, shown in Figure 3.1, indicating that the data was not normally distributed.

This could be due in part to the artificial IRI minimum of $0.06 \mathrm{~s}$, which was enforced to exclude intervals which are physiologically impossible. In addition, the Bruesch-Pagan test revealed that IRI data were not homoscedastic $(p=0.045)$. Because of this right skew and failure to meet standards of homoscedasticity, the IRI data could not satisfy the assumptions of linear regression. To improve the model, a nonlinear transformation was applied by using the reciprocal of the mean IRI, which in this case is the physiologically relevant measure of rate. Again, a QQ plot and the Breusch-Pagan test were employed, revealing that rate was homoscedastic $(\mathrm{p}=0.056)$ and better approximated a normal distribution as seen in Figure 3.2B.

Therefore, we determined that rate was a better candidate for linear regression and used rate as the response variable rather than mean IRI. This model revealed no significant effect of strain on average rate $(p=0.088$, adjusted for sex), so strain was removed from the model to improve fit. Female animals showed significantly slower respiratory rates ( $\mathrm{p}$ $=0.019$ ) than males, independent of strain. This result was opposite to what we predicted in terms of sex, based on Liu et al. 2020 [28]. Raw residuals were plotted against fitted values, shown in Figure 3.2C, which revealed that female animals had greater residual variability than males. This can also be seen in the spread of rate values as shown in the boxplot in Figure 3.2A. Regardless, the effect of sex was modest $(\beta=-0.817)$ and average rates were $6.81 \mathrm{~Hz}$ for females and $5.99 \mathrm{~Hz}$ for males. Our hypothesis was supported, however, in that conditional loss of $E n 1 / 2$ in the $\mathrm{eCN}$ did not have a significant effect on average respiratory rate. To be sure that the sex difference in rate did not influence $\mathrm{CV}$ or $\mathrm{CV} 2$, rate was initially included as a predictor variable in the remaining linear regressions. 

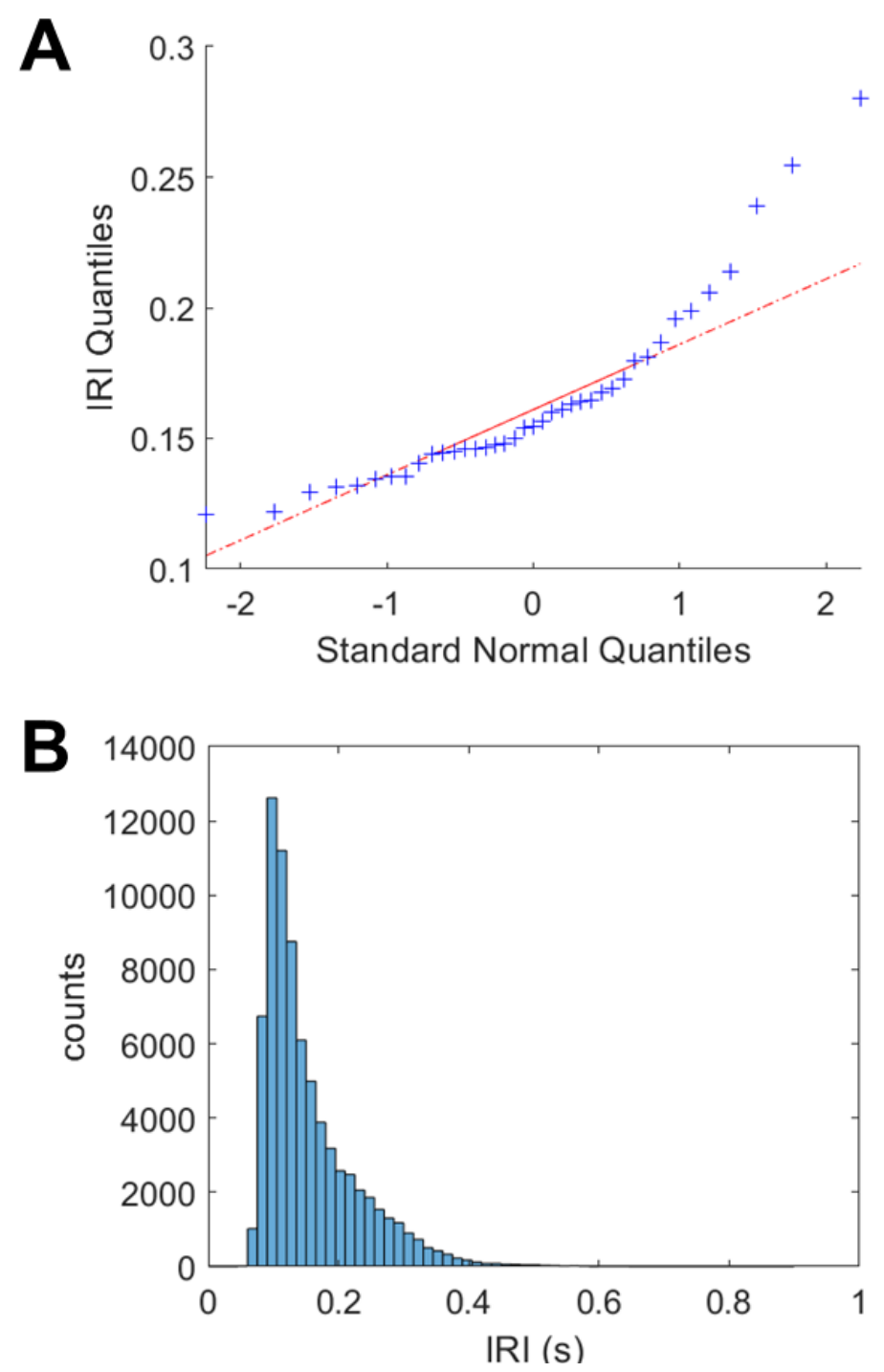

Figure 3.1: IRI data do not fit assumptions of linear regression. A) Quantile-quantile plot of average IRI data versus standard normal distribution reveals deviation from normality. Blue plus signs indicate quantiles, dotted red line represents the plot of the line if data has a perfectly normal distribution. B) Histogram of total IRI data (not averages) with a bin size of 0.015 seconds. The right skew and artificial cutoff at 0.06 seconds can be clearly seen. 
A

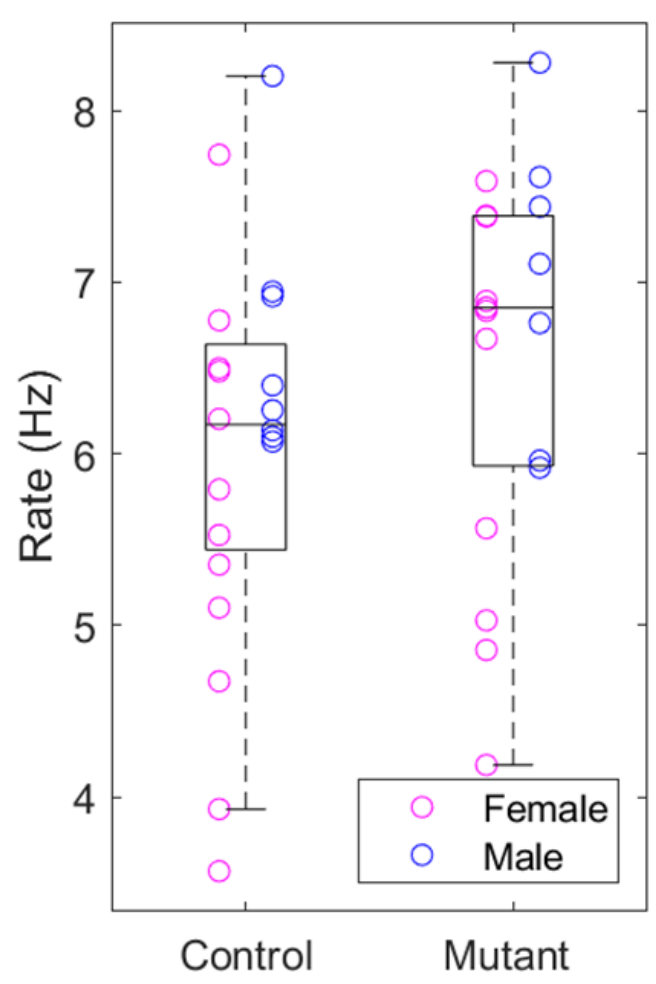

B
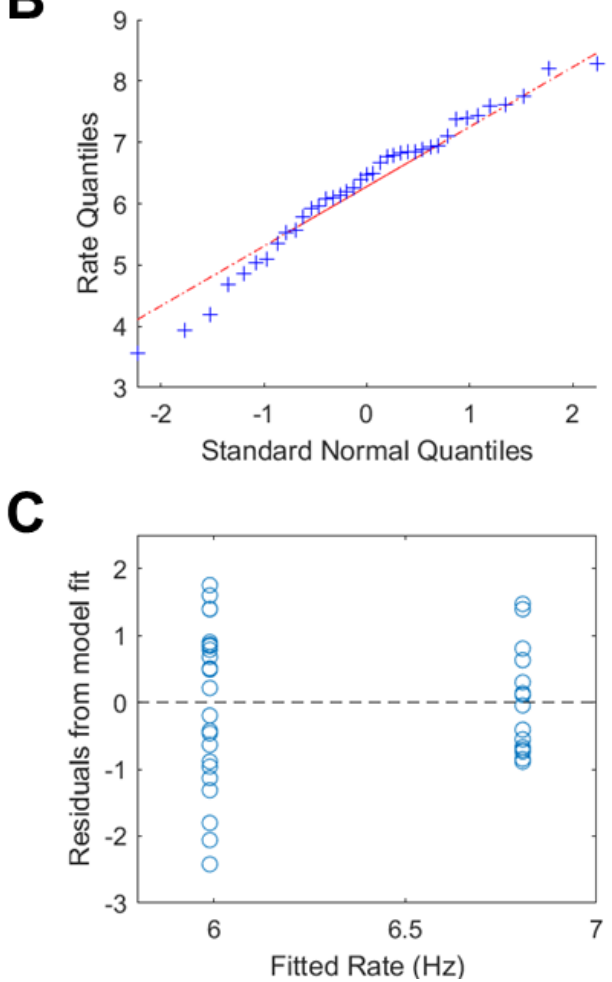

Figure 3.2: Average respiratory rate is influenced by sex and not strain. A) Boxplot showing the average respiratory rates of individual mice (open circles). There was no significant effect of strain on respiratory rate ( $\mathrm{p}=$ 0.088 , adjusted for sex). After strain was removed from model, sex remained a significant predictor of average rate $(\mathrm{p}=0.019)$. The upper, middle, and lower box limits indicate the 75th percentile, median, and 25th percentile, respectively. The whiskers represent $\pm 2.7 \sigma$, encompassing $99.3 \%$ of the data if the data are normally distributed. B) Quantile-quantile plot of rate data versus standard normal distribution. Blue plus signs indicate quantiles, dotted red line represents the plot of the line if data has a perfectly normal distribution. C) Plot of residuals versus fitted rate, based on the linear model with sex as the only predictor of rate. Ideally, residuals are distributed equally for both males and females with no clear trend. Actual data shows an apparent increased spread of residuals for female data as compared to males. Fitted rate values: all female, $5.99 \mathrm{~Hz}$; all male, $6.81 \mathrm{~Hz}$. 
Rate was not significant in either case ( $\mathrm{p}>0.05$, data not shown) so it was removed from the models.

\subsection{Mice with Conditional Loss of En1/2 in Excitatory Cerebellar Neurons Show Decreased Overall Respiratory Variability}

Next, we investigated if the conditional loss of $E n 1 / 2$ in eCN affects eupneic respiratory variability using the coefficient of variation of IRIs. The QQ plot revealed that CV values were basically normally distributed (Figure 3.3B) and Breusch-Pagan test confirmed homoscedasticity $(\mathrm{p}=0.66)$.

The residual plot in Figure 3.3C further confirmed appropriate model fit as there was no apparent trend to the residuals. There was a significant effect of both sex and strain on $C V(p=0.0036$ and $p=0.021$, respectively), with mutants animals having decreased CV as compared to control littermates, and males having decreased CV compared to females as can be seen in Figure 3.3A. In contrast to our expectations, this indicated that conditional loss of $E n 1 / 2$ in eCN significantly decreased overall respiratory variability during eupnea, and indicates decreased variability in males compared to females.

\subsection{Mice with Conditional Loss of En1/2 in Excitatory Cerebellar Neurons Show Increased Intrinsic Respiratory Rhythmicity}

Finally, we sought to evaluate the effect of conditional loss of En1/2 in the eCN on intrinsic respiratory rhythmicity during eupnea. As with rate and $\mathrm{CV}, \mathrm{CV} 2$ data was also confirmed to be normal (QQ plot in Figure 3.4B) and homoscedastic ( $\mathrm{p}=0.29$, Breusch-Pagan test), and there was no pattern to the residual plot (Figure 3.4C).

Similar to $\mathrm{CV}$, linear regression of $\mathrm{CV} 2$ revealed a significant effect of both sex and strain (Figure 3.4A). Mutant animals had significantly decreased CV2 values compared to control littermates ( $\mathrm{p}<<0.001$ ) and males had significantly decreased CV2 values compared to females $(\mathrm{p}=0.0025)$. In other words, conditional loss of $E n 1 / 2$ in the eCN resulted in increased intrinsic respiratory rhythmicity during eupnea. 

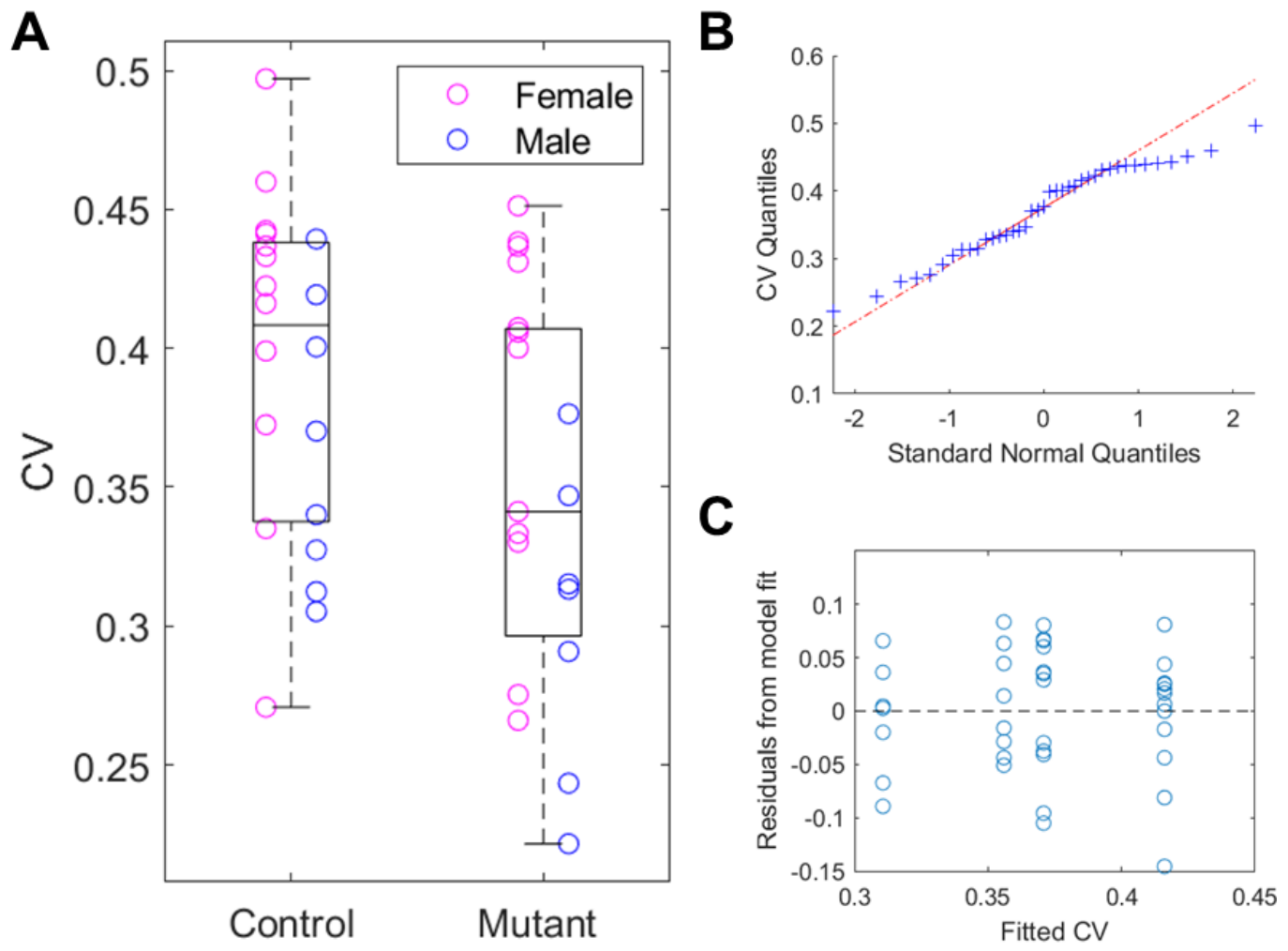

Figure 3.3: CV of IRI distribution is influenced by both sex and strain. A) Boxplot showing the CV of the IRI distribution of individual mice (open circles). Linear regression revealed a significant effect of both sex ( $\mathrm{p}=$ $0.0036)$ and strain $(p=0.021)$ on $C V$. The upper, middle, and lower box limits indicate the 75th percentile, median, and 25th percentile, respectively. The whiskers represent $\pm 2.7 \sigma$, encompassing $99.3 \%$ of the data if the data are normally distributed. B) Quantile-quantile plot of CV data versus standard normal distribution. Blue plus signs indicate quantiles, dotted red line represents the plot of the line if data has a perfectly normal distribution. C) Plot of residuals versus fitted CV. The four groups represented in fitted values are due to the fact that there can only be four possible combinations of the predictors sex and strain. Ideally, residuals are distributed equally amongst groups with no clear trend. Actual data shows no obvious trend, indicating good model fit. Fitted CV values: male mutant, 0.311 ; male control, 0.356 ; female mutant, 0.371 ; female control, 0.416 . 
A

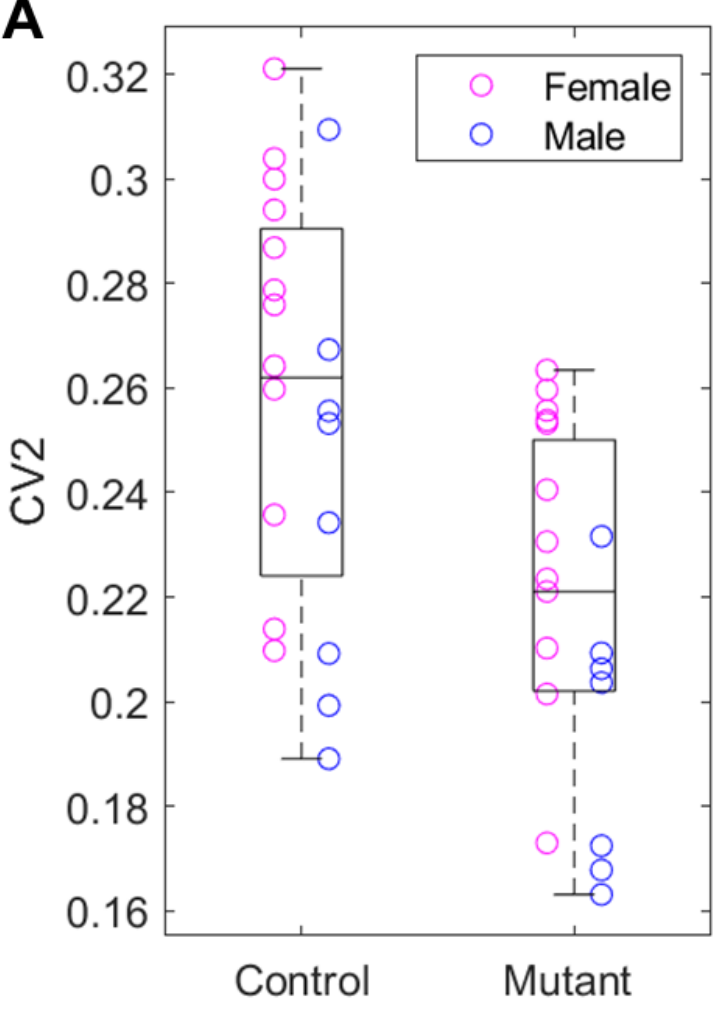

B

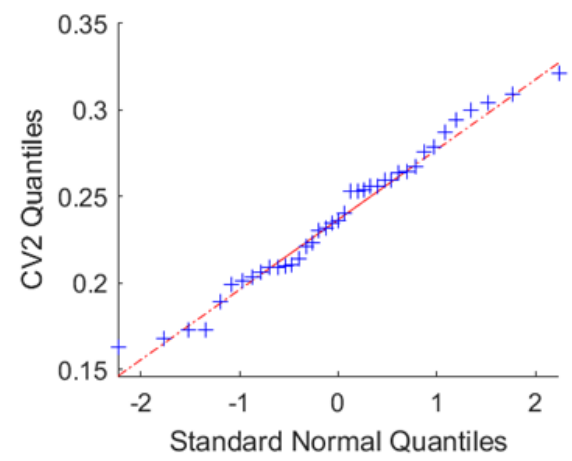

C

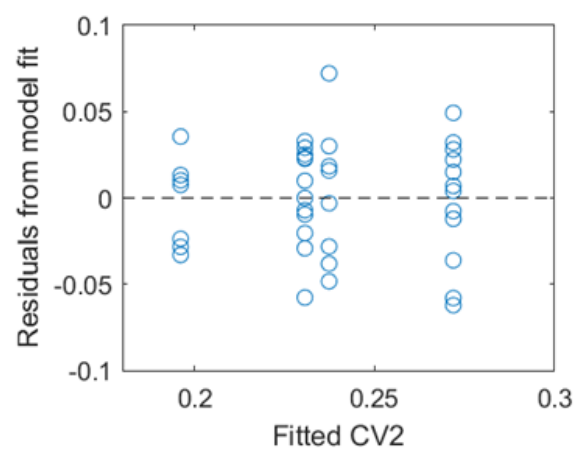

Figure 3.4: Mean CV2 is influenced by both sex and strain. A) Boxplot showing the mean CV2 of individual mice (open circles). Linear regression revealed a significant effect of both sex $(p=0.0025)$ and strain $(p<0.001)$ on CV2. The upper, middle, and lower box limits indicate the 75th percentile, median, and 25th percentile, respectively. The whiskers represent $\pm 2.7 \sigma$, encompassing $99.3 \%$ of the data if the data are normally distributed. B) Quantile-quantile plot of CV2 data versus standard normal distribution. Blue plus signs indicate quantiles, dotted red line represents the plot of the line if data has a perfectly normal distribution. C) Plot of residuals versus fitted CV2. The four groups represented in fitted values are due to the fact that there can only be four possible combinations of the predictors, sex and strain. Ideally, residuals are distributed equally amongst groups with no clear trend. Actual data shows no obvious trend, indicating good model fit. Fitted CV2 values: male mutant, 0.196; male control, 0.237; female mutant, 0.231; female control, 0.272 . 


\section{Chapter 4}

\section{Discussion}

Based on our previous studies which demonstrated the importance of the cerebellar cortex during eupneic breathing [28] and established evidence for a relationship between the medial DCN and respiration [30], we expected that animals with conditional loss of En1/2 in excitatory cerebellar neurons would show altered eupneic respiratory rhythmicity compared to controls. This study confirmed our expectations in that mutant animals had significantly increased intrinsic respiratory rhythmicity, as evidenced by a decrease in CV2. These results mirrored Liu et al., where Purkinje cell silencing also caused decreased CV2 [28]. As predicted, we also found no difference between mutant Atoh1-En1/2 animals and their control littermates in average respiratory rate. However, this present study diverged from expectations about the distribution of IRIs as measured by CV. While we hypothesized no change in IRI variability or CV, we found that mutant Atoh1-En1/2 animals exhibited decreased overall variability during eupnea. Finally, we found sex differences in all three response variables, with females having increased CV and CV2 values and decreased average respiratory rate as compared to males, irrespective of strain. A modest effect of sex was also found in Liu et al. for both respiratory rate and CV2, although no effect of sex was found for $\mathrm{CV}$ in that study. In humans, there is broad evidence of physiological differences in respiration between the sexes, but these have been mainly attributed to the average size difference between male and female bodies [29]. For example, smaller lung size in females may lead to smaller tidal volume than males, requiring a faster respiratory rate to compensate. In contrast, our results showed a slower respiratory rate in females, but we cannot say whether this was due to differences in tidal volume because it was not measured. In a study in developing rats (neonatal to young adult, P10-P90), Holley et al. found that both age and sex affected respiratory responses to hypoxia, hypercapnia, and normoxia [21]. Most relevant to this study, they found that male P12-13 rats (in the postnatal "sensitive period") had significantly decreased minute ventilation and tidal volume in normoxic conditions as compared to females. This also does not account for the sex differences in $\mathrm{CV}$ or $\mathrm{CV} 2$, but these results certainly indicate that future studies of eupneic respiration should continue to include sex as factor. In addition, given that the Atoh1-En1/2 CKO strain affects cerebellar development specifically, future research with this model should repeat wholebody unrestrained plethysmography at different developmental time points to understand how mutant respiratory response changes with age and sex. 
The proposed role of the cerebellum in coordinating respiratory and other orofacial movements has been investigated in both mice and humans, and the vast reciprocal connections between the cerebellum and brainstem provide a putative anatomical substrate $[42,4,18,5,30,62]$. Inappropriate coordination of swallowing and respiration can have detrimental effects, as in aspiration pneumonia due to dysphagia in patients with cerebellar ataxia [42]. In our recently published paper by, we sought to artificially "restore" appropriate cerebellar coordination by introducing intrinsic rhythmicity into the respiratory sequence of mutant animals with Purkinje cell silencing [28]. In this model, we altered the true mutant respiratory sequence in silico by lengthening every 10 th IRI by $50 \%$ (about $75 \mathrm{~ms}$ ). With this change, there was no longer a significant difference between the CV2 of the altered respiratory sequence and that of control animals. In addition, this alteration did not introduce a significant difference in CV between mutants and controls. This suggested that the CRB may have a role in altering the length of select respiratory intervals, and we speculated that this pause in respiration could be induced by the CRB to allow time for swallowing. Future studies should take care to include additional behavioral measures such as licking, swallowing, and whisking in order to develop a more complete understanding. These studies could also dissect breathing at rest from sniffing behaviors, as this present work considered eupneic respiration as whole.

The Atoh1-En1/2 CKO strain provides unique insight into cerebellar temporal coordination. The recent consensus paper by Bareš et al. emphasized three important aspects of the cerebellum which make it suited for its timekeeping role: microcircuitry, neuronal density, and vast extracerebellar interconnectivity [1]. Willett et al. reported that this mutant model has normal neuronal density, despite its proportionally decreased neuronal number in certain hypoplastic lobules [53]. It is generally accepted that PCs in the cerebellar cortex project to the DCN which is anatomically closest to them [26], and accordingly, Willet et al. showed that PCs in the affected hypoplastic lobules projected to the medial and intermediate DCN, which were also reduced in size. This suggests that while these structures are reduced in size, the projection patterns from PCs to DCN are likely maintained. Therefore, we suggest that the altered respiratory rhythmicity is due to an error in the third essential aspect of cerebellar design - its highly organized and extensive extracerebellar interconnectivity.

While microcircuitry remains largely unchanged throughout the healthy cerebellar cortex, the discovery of molecular parasagittal stripes and functional connectivity mapping have revealed that the cerebellum is organized into spatially distinct zones [37, 6]. In 2008, Sillitoe et al. reported that Engrailed 1 and 2 are not only required for normal foliation and molecular patterning of the cerebellum, but that they are also involved in mossy fiber afferent targeting [47]. This paper also suggested that morphological defects do not necessarily imply circuit defects. Instead, they urged that a better predictor of improper circuitry is an aberrant molecular pattern. In a mouse strain with conditional loss of En1/2 in the whole cerebellum except for PCs, foliation defects were present but the molecular pattern and circuitry were intact. Thus the authors posited that PC expression of En1/2 was required for guiding mossy fiber afferents. It also important to note that while the 
Atoh1-En1/2 CKO strain affects cerebellar granule cells and the $\mathrm{eCN}$, it is unknown what effect conditional loss of En1/2 in other rhombic lip-lineage structures, such as certain precerebellar nuclei, may have on respiration. Although the recent study by Liu et al. showed that CRB-specific mutations are sufficient to alter the CV2 of eupneic respiration [28], it does not preclude the possibility that extracerebellar loss of En1/2 could also influence the $\mathrm{CV}$ and CV2 of eupneic respiration. I suggest that future research focus on further characterizing the Atoh1-En1/2 CKO model, evaluating potential changes to the cerebellar molecular pattern and circuitry, as well as investigating potentially affected extracerebellar structures, to better understand how these factors may influence the cerebellar role in eupneic respiratory patterns. 


\section{List of References}

[1] Martin Bareš et al. "Consensus paper: Decoding the Contributions of the Cerebellum as a Time Machine. From Neurons to Clinical Applications". In: The Cerebellum 18.2 (Apr. 2019), pp. 266-286. ISSN: 1473-4230. DOI: 10.1007/s12311-018-0979-5.

[2] M. Bassal and A. L. Bianchi. "Inspiratory onset or termination induced by electrical stimulation of the brain". In: Respir Physiol 50.1 (Oct. 1982), pp. 23-40. ISSN: 0034-5687 (Print) 0034-5687 (Linking).

[3] T. S. Breusch and A. R. Pagan. "A Simple Test for Heteroscedasticity and Random Coefficient Variation". In: Econometrica 47.5 (1979), pp. 1287-1294. ISSN: 00129682, 14680262. DOI: $10.2307 / 1911963$.

[4] J. L. Bryant et al. "A proposed new function of the mouse cerebellum: Temporal modulation of brain stem pattern generator activity". In: Soc.Neurosci.Abstr. 78.17 (2007).

[5] J. L. Bryant et al. "Cerebellar cortical output encodes temporal aspects of rhythmic licking movements and is necessary for normal licking frequency". eng. In: Eur J Neurosci 32.1 (July 2010), pp. 41-52. ISSN: 1460-9568 (Electronic) 0953-816X (Linking). DOI: EJN7244 [pii] 10.1111/j.1460-9568.2010.07244.x.

[6] R. L. Buckner et al. "The organization of the human cerebellum estimated by intrinsic functional connectivity". In: Journal of Neurophysiology 106.5 (Nov. 2011), pp. 2322-45. ISSN: 1522-1598 (Electronic) 0022-3077 (Linking). DOI: 10.1152/jn. 00339. 2011.

[7] E. K. Bystrzycka and B. S. Nail. "The source of the respiratory drive to nasolabialis motoneurones in the rabbit; a HRP study". eng. In: Brain Res 266.2 (May 1983), pp. 183-91. ISSN: 0006-8993 (Print) 0006-8993. DOI: 10.1016/0006-8993 (83) 90648-0.

[8] M. A. Calton et al. "The Cerebellum and SIDS: Disordered Breathing in a Mouse Model of Developmental Cerebellar Purkinje Cell Loss during Recovery from Hypercarbia". In: Front Neurol 7 (2016), p. 78. ISSN: 1664-2295 (Electronic) 1664-2295 (Linking). DOI: 10.3389/fneur . 2016.00078.

[9] M. L. Chen et al. "Disordered respiratory control in children with partial cerebellar resections". In: Pediatr.Pulmonol. 40.1 (2005), pp. 88-91.

[10] Y. Cheng et al. "The Engrailed homeobox genes determine the different foliation patterns in the vermis and hemispheres of the mammalian cerebellum". eng. In: Development 137.3 (Feb. 2010), pp. 519-29. ISSN: 0950-1991 (Print) 0950-1991. DOI: $10.1242 / \mathrm{dev} .027045$.

[11] C. A. Davis and A. L. Joyner. "Expression patterns of the homeo box-containing genes En-1 and En-2 and the proto-oncogene int-1 diverge during mouse development". In: Genes Dev. 2.12B (1988), pp. 1736-1744. 
[12] E. G. Dobbins and J. L. Feldman. "Brainstem network controlling descending drive to phrenic motoneurons in rat". eng. In: J Comp Neurol 347.1 (Sept. 1994), pp. 64-86. ISSN: 0021-9967 (Print) 0021-9967. DOI: 10.1002/cne.903470106.

[13] J. L. Feldman and C. A. Del Negro. "Looking for inspiration: new perspectives on respiratory rhythm". In: Nat.Rev.Neurosci 7.3 (2006), pp. 232-242.

[14] Kevin Flurkey, Joanne M. Currer, and D. E. Harrison. "Chapter 20 - Mouse Models in Aging Research". In: The Mouse in Biomedical Research (Second Edition). Ed. by James G. Fox et al. Burlington: Academic Press, Jan. 2007, pp. 637-672. ISBN: 978-012-369454-6.

[15] B. J. Fredette and E. Mugnaini. "The GABAergic cerebello-olivary projection in the rat". eng. In: Anat Embryol (Berl) 184.3 (1991), pp. 225-43. ISSN: 0340-2061 (Print) 0340-2061. DOI: 10.1007/bf01673258.

[16] D. Gozal et al. "Localization of putative neural respiratory regions in the human by functional magnetic resonance imaging". eng. In: J Appl Physiol (1985) 76.5 (May 1994), pp. 2076-83. ISSN: 8750-7587 (Print) 0161-7567. DOI: 10.1152/jappl .1994. 76. 5.2076.

[17] A. Gruart and J. M. Delgado-Garcia. "Respiration-related neurons recorded in the deep cerebellar nuclei of the alert cat". eng. In: Neuroreport 3.4 (Apr. 1992), pp. 365-8. ISSN: 0959-4965 (Print) 0959-4965 (Linking).

[18] Anna I. Hårdemark Cedborg et al. "Co-ordination of spontaneous swallowing with respiratory airflow and diaphragmatic and abdominal muscle activity in healthy adult humans". In: Experimental Physiology 94.4 (Apr. 2009), pp. 459-468. ISSN: 09580670. DOI: 10.1113/expphysiol.2008.045724.

[19] R. M. Harper et al. "Regional brain activation in humans during respiratory and blood pressure challenges". In: Clin.Exp.Pharmacol.Physiol 25.6 (1998), pp. 483-486.

[20] Meike E. van der Heijden and Huda Y. Zoghbi. "Loss of Atoh1 from neurons regulating hypoxic and hypercapnic chemoresponses causes neonatal respiratory failure in mice". eng. In: eLife 7 (2018), e38455. ISSN: 2050-084X. DOI: 10 .7554/eLife . 38455.

[21] Heidi S. Holley, Mary Behan, and Julie M. Wenninger. "Age and sex differences in the ventilatory response to hypoxia and hypercapnia in awake neonatal, pre-pubertal and young adult rats". In: Respiratory Physiology \& Neurobiology 180.1 (Jan. 2012), pp. 79-87. ISSN: 1569-9048. DOI: 10.1016/j . resp. 2011 .10.012. URL: http: //www . sciencedirect.com/science/article/pii/S1569904811003624.

[22] G. R. Holt et al. "Comparison of discharge variability in vitro and in vivo in cat visual cortex neurons". In: Journal of Neurophysiology 75.5 (May 1996), pp. 1806-14. ISSN: 0022-3077 (Print) 0022-3077 (Linking).

[23] Q. Huang, D. Zhou, and W. M. St John. "Cerebellar control of expiratory activities of medullary neurons and spinal nerves". In: J.Appl.Physiol 74.4 (1993), pp. 1934-1940.

[24] W. H. Huang et al. "Atoh1 governs the migration of postmitotic neurons that shape respiratory effectiveness at birth and chemoresponsiveness in adulthood". In: Neuron 75.5 (Sept. 2012), pp. 799-809. ISSN: 1097-4199 (Electronic) 0896-6273 (Linking). DOI: $10.1016 / j$.neuron. 2012.06.027. 
[25] E. Legue et al. "Differential timing of granule cell production during cerebellum development underlies generation of the foliation pattern". In: Neural Dev 11.1 (Sept. 2016), p. 17. ISSN: 1749-8104 (Electronic) 1749-8104 (Linking). DOI: 10.1186/s13064016-0072-z.

[26] K. Leto et al. "Consensus Paper: Cerebellar Development". In: Cerebellum 15.6 (Dec. 2016), pp. 789-828. ISSN: 1473-4230 (Electronic) 1473-4222 (Linking). DOI: 10 . $1007 /$ s12311-015-0724-2.

[27] R. Lim et al. "Measuring respiratory function in mice using unrestrained wholebody plethysmography". In: J Vis Exp 90 (2014), e51755. ISSN: 1940-087X (Electronic) 1940-087X (Linking). DOI: 10.3791/51755.

[28] Yu Liu et al. "Loss of cerebellar function selectively affects intrinsic rhythmicity of eupneic breathing". eng. In: Biology open 9.4 (2020), bio048785. ISSN: 2046-6390. DOI: 10.1242/bio. 048785.

[29] Antonella LoMauro and Andrea Aliverti. "Sex differences in respiratory function". eng. In: Breathe (Sheffield, England) 14.2 (June 2018). Publisher: European Respiratory Society, pp. 131-140. ISSN: 1810-6838. DOI: 10 .1183/20734735.000318. URL: https : //pubmed.ncbi.nlm.nih.gov/29875832.

[30] L. Lu et al. "Medial cerebellar nuclear projections and activity patterns link cerebellar output to orofacial and respiratory behavior." In: Front. Neural Circuits 7:56 (2013).

[31] P. M. Macey et al. "Hypoxia reveals posterior thalamic, cerebellar, midbrain, and limbic deficits in congenital central hypoventilation syndrome". In: J.Appl.Physiol 98.3 (2005), pp. 958-969.

[32] Rob Machold and Gord Fishell. "Math1 Is Expressed in Temporally Discrete Pools of Cerebellar Rhombic-Lip Neural Progenitors". In: Neuron 48.1 (Oct. 2005), pp. 17-24. ISSN: 0896-6273. DOI: https://doi .org/10.1016/j . neuron. 2005.08.028.

[33] Martin Magris. Breusch-Pagan Test. 2020.

[34] Frederick R. Miller. “THE PHYSIOLOGY OF THE CEREBELLUM". In: Physiological Reviews 6.1 (Jan. 1926), pp. 124-159. ISSN: 0031-9333. DOI: 10.1152/physrev . 1926. 6.1 .124$.

[35] Giuseppe Moruzzi. "PALEOCEREBELLAR INHIBITION OF VASOMOTOR AND RESPIRATORY CAROTID SINUS REFLEXES". In: Journal of Neurophysiology 3.1 (Jan. 1940), pp. 20-32. ISSN: 0022-3077. DOI: 10.1152/jn.1940.3.1.20.

[36] R. M. Napper and R. J. Harvey. "Number of parallel fiber synapses on an individual Purkinje cell in the cerebellum of the rat". eng. In: J Comp Neurol 274.2 (Aug. 1988), pp. 168-77. ISSN: 0021-9967 (Print) 0021-9967. DOI: 10.1002/cne. 902740204.

[37] J. X. O'Reilly et al. "Distinct and overlapping functional zones in the cerebellum defined by resting state functional connectivity". In: Cereb Cortex 20.4 (Apr. 2010), pp. 953-65. ISSN: 1460-2199 (Electronic) 1047-3211 (Linking). DOI: 10.1093/cercor/ bhp157.

[38] G. D. Orvis et al. "The engrailed homeobox genes are required in multiple cell lineages to coordinate sequential formation of fissures and growth of the cerebellum". In: Dev Biol 367.1 (July 2012), pp. 25-39. ISSN: 1095-564X (Electronic) 0012-1606 (Linking). DOI: $10.1016 / j \cdot y d b i o .2012 .04 .018$. 
[39] L. M. Parsons et al. "Neuroimaging evidence implicating cerebellum in the experience of hypercapnia and hunger for air". In: Proc.Natl.Acad.Sci.U.S.A 98.4 (2001), pp. 2041-2046.

[40] J. F. Paton et al. "Efferent connections of lobule IX of the posterior cerebellar cortex in the rabbit-some functional considerations". eng. In: J Auton Nerv Syst 36.3 (Dec. 1991), pp. 209-24. ISSN: 0165-1838 (Print) 0165-1838. DOI: 10 . 1016/0165-1838 (91) 90045-5.

[41] D. Purves et al., eds. Neuroscience. 2nd. Sunderland, MA: Sinauer Associates, 2001. ISBN: 0-87893-742-0.

[42] L. Ramio-Torrentia, E. Gomez, and D. Genis. "Swallowing in degenerative ataxias". In: J.Neurol. 253.7 (2006), pp. 875-881.

[43] D. M. Rector, C. A. Richard, and R. M. Harper. "Cerebellar fastigial nuclei activity during blood pressure challenges". In: J.Appl.Physiol 101.2 (2006), pp. 549-555.

[44] M. V. Sergievskii and NIa Kireeva. "[Reciprocal connections of respiratory center nuclei]". rus. In: Biull Eksp Biol Med 90.12 (Dec. 1980), pp. 652-4. ISSN: 0365-9615 (Print) 0365-9615.

[45] R. V. Sillitoe and A. L. Joyner. "Morphology, molecular codes, and circuitry produce the three-dimensional complexity of the cerebellum". In: Annu Rev Cell Dev Biol 23 (2007), pp. 549-77. ISSN: 1081-0706 (Print) 1081-0706 (Linking). DOI: 10 . 1146/ annurev.cellbio.23.090506.123237.

[46] R. V. Sillitoe, M. W. Vogel, and A. L. Joyner. "Engrailed homeobox genes regulate establishment of the cerebellar afferent circuit map". In: J. Neurosci. 30.30 (2010), pp. 10015-10024. DOI: 10.1101/310953.

[47] R. V. Sillitoe et al. "Engrailed homeobox genes determine the organization of Purkinje cell sagittal stripe gene expression in the adult cerebellum". eng. In: J Neurosci 28.47 (Nov. 2008), pp. 12150-62. ISSN: 0270-6474 (Print) 0270-6474. DOI: 10 . 1523 / jneurosci.2059-08.2008.

[48] Martha L. Streng, Laurentiu S. Popa, and Timothy J. Ebner. "Complex Spike Wars: a New Hope". In: The Cerebellum 17.6 (Dec. 2018), pp. 735-746. ISSN: 1473-4230. DOI: 10.1007/s12311-018-0960-3.

[49] M. Uusisaari and E. De Schutter. "The mysterious microcircuitry of the cerebellar nuclei". eng. In: J Physiol 589.Pt 14 (July 2011), pp. 3441-57. ISSN: 0022-3751 (Print) 0022-3751. DOI: 10.1113/jphysiol.2010.201582.

[50] Vincent Y. Wang, Matthew F. Rose, and Huda Y. Zoghbi. "Math1 Expression Redefines the Rhombic Lip Derivatives and Reveals Novel Lineages within the Brainstem and Cerebellum". In: Neuron 48.1 (Oct. 2005), pp. 31-43. ISSN: 0896-6273. DOI: https: //doi.org/10.1016/j.neuron.2005.08.024.

[51] D. W. Wesson et al. "Respiratory and sniffing behaviors throughout adulthood and aging in mice". eng. In: Behavioural brain research 223.1 (Sept. 2011), pp. 99-106. ISSN: 1872-7549 (Electronic) 0166-4328 (Linking). DOI: 10.1016/j . bbr .2011 .04 . 016.

[52] D. W. Wesson et al. "Sniffing behavior of mice during performance in odor-guided tasks". eng. In: Chemical senses 33.7 (Sept. 2008), pp. 581-96. ISSN: 1464-3553 (Electronic) 0379-864X (Linking). DOI: 10.1093/chemse/bjn029. 
[53] Ryan T. Willett et al. "Cerebellar nuclei excitatory neurons regulate developmental scaling of presynaptic Purkinje cell number and organ growth". eng. In: eLife 8 (2019), e50617. ISSN: 2050-084X. DOI: 10.7554/eLife.50617.

[54] John L. Williams, S. J. Everse, and L. O. Lutherer. "Stimulating fastigial nucleus alters central mechanisms regulating phrenic activity". In: Respir Physiol 76.2 (May 1989), pp. 215-227. ISSN: 0034-5687. DOI: https : / / doi . org / 10 . 1016/0034-5687 (89) 90099-6.

[55] S. L. Wilson et al. "Spatially restricted and developmentally dynamic expression of engrailed genes in multiple cerebellar cell types". In: Cerebellum 10.3 (Sept. 2011), pp. 356-72. ISSN: 1473-4230 (Electronic) 1473-4222 (Linking). DOI: $10.1007 /$ s12311011-0254-5.

[56] Richard Wingate. "Math-Map(ic)s". In: Neuron 48.1 (Oct. 2005), pp. 1-4. ISSN: 08966273. DOI: https://doi.org/10.1016/j.neuron.2005.09.012.

[57] F. Xu and D. T. Frazier. "Involvement of the fastigial nuclei in vagally mediated respiratory responses". eng. In: J Appl Physiol (1985) 82.6 (June 1997), pp. 1853-61. ISSN: 8750-7587 (Print) 0161-7567. DOI: 10.1152/jappl .1997.82.6.1853.

[58] F. Xu and D. T. Frazier. "Medullary respiratory neuronal activity modulated by stimulation of the fastigial nucleus of the cerebellum". In: Brain Res 705.1-2 (Dec. 1995), pp. 53-64. ISSN: 0006-8993 (Print) 0006-8993 (Linking).

[59] F. Xu and D. T. Frazier. "Modulation of respiratory motor output by cerebellar deep nuclei in the rat". In: J.Appl.Physiol 89.3 (2000), pp. 996-1004.

[60] F. Xu and D. T. Frazier. "Role of the cerebellar deep nuclei in respiratory modulation". In: Cerebellum. 1.1 (2002), pp. 35-40.

[61] F. Xu, J. Owen, and D. T. Frazier. "Hypoxic respiratory responses attenuated by ablation of the cerebellum or fastigial nuclei". eng. In: J Appl Physiol (1985) 79.4 (Oct. 1995), pp. 1181-9. ISSN: 8750-7587 (Print) 0161-7567. DOI: 10 .1152/ jappl . 1995 . 79. 4.1181.

[62] Naomi Yagi et al. "Inappropriate Timing of Swallow in the Respiratory Cycle Causes Breathing-Swallowing Discoordination". English. In: Frontiers in Physiology 8.676 (Sept. 2017). ISSN: 1664-042X. DOI: 10.3389/fphys.2017.00676.

[63] Z. Zhang, F. Xu, and D. T. Frazier. "Role of the Botzinger complex in fastigial nucleusmediated respiratory responses". In: Anat Rec 254.4 (Apr. 1999), pp. 542-8. ISSN: 0003-276X (Print) 0003-276X (Linking). 


\section{Vita}

Angela P. Taylor was born in 1995 to Douglas and Constance Taylor in Huntsville, AL. After receiving her high school diploma from Covenant Christian Academy, she began her undergraduate education at Union University in Jackson, TN. There she received her Bachelor of Science in Biochemistry with a minor in Psychology. She graduated summa cum laude in May 2017 and then matriculated into the Biomedical Sciences program at the University of Tennessee Health Science Center, Memphis, TN. In September 2020, she expects to receive the Master of Science degree in Biomedical Sciences with a concentration in Neuroscience from the University of Tennessee. 